\title{
ESD with double clips and rubber band traction of neoplastic lesions developed in the appendiceal orifice is effective and safe
}

\section{(ㄷ)(우우}

\author{
Authors \\ Borathchakra Oung ${ }^{1,2}$, Jérôme Rivory ${ }^{1}$, Edouard Chabrun ${ }^{3}$, Romain Legros ${ }^{4}$, Julien Faller ${ }^{1}$, Florence Léger-Nguyen ${ }^{5}$, \\ Florian Rostain ${ }^{1}$, Charles-Eric Ber ${ }^{6}$, Valérie Hervieu ${ }^{7}$, Jean-Christophe Saurin ${ }^{1}$, Thierry Ponchon ${ }^{1}$, Jérémie Jacques ${ }^{4}$, \\ Mathieu Pioche ${ }^{1}$
}

Institutions

1 Department of Endoscopy and Gastroenterology, Pavillon L, Edouard Herriot Hospital, Hospices Civils de Lyon, Lyon, France

2 Cambodian Association of Gastrointestinal Endoscopy (CAGE), Cambodia

3 Department of Endoscopy and Gastroenterology, Bordeaux University Hospital, Bordeaux, France

4 Department of Endoscopy and Gastroenterology, Dupuytren University Hospital, Limoges, France

5 Gastroenterology and Endoscopy, Val d'ouest private clinic, Ecully, France

6 Department of Anesthesiology, Pavillon L, Edouard Herriot Hospital, Hospices Civils de Lyon, Lyon, France

7 Department of Pathology, Pavillon L, Edouard Herriot Hospital, Hospices Civils de Lyon, Lyon, France

submitted 1.7.2019

accepted after revision 17.11.2019

\section{Bibliography}

DOI https://doi.org/10.1055/a-1072-4830 |

Endoscopy International Open 2020; 08: E388-E395

(c) Georg Thieme Verlag KG Stuttgart · New York elSSN 2196-9736

Corresponding author

Dr. Mathieu Pioche, Endoscopy unit - Digestive Disease

department, Pavillon L - Edouard Herriot Hospital, 69437

Lyon Cedex, France

Fax: +0472110146

mathieu.pioche@chu-lyon.fr

\section{ABSTRACT}

Background and study aims Endoscopic submucosal dissection (ESD) of superficial colorectal lesions in close proximity to the appendiceal orifice (L-PAO) was shown to be feasible except in case of deep invasion into the appendix (type 3 of Toyonaga's classification). This study aimed to determine the outcomes of ESD with double clip and rubber band traction (DCT-ESD) of L-PAO including a majority of type 3.

Patients and methods We reviewed retrospectively all consecutive DCT-ESD of L-PAO performed in 3 French centers. Each lesion was described according to Toyonaga's classification and type 0 lesions were excluded. The primary outcome was en bloc and R0 resection rates for L-PAO. Morbidity and salvage surgery were recorded.

Results A total of 32 patients underwent DCT-ESD; 22 lesions $(68.8 \%)$ were type 3 , including 11 with previous appendectomy (34.4\%). Median lesion size was $35 \mathrm{~mm}$ range $(10-110 \mathrm{~mm})$ and median duration of resection was $47 \mathrm{~min}$ range (10-230 min). We achieved $100 \%$ of En bloc resection exclusively with DCT-ESD and $90.6 \%$ of histological R0 resection rate. Per-procedure, 11 perforations occurred and were all immediately closed with clips. Overall, 3 patients (10.7\%) underwent surgery without stoma (2 complications related and 1 incomplete resection). No death occurred.

Conclusion ESD of lesions deeply invading appendiceal orifice is feasible with the help of a traction system. Technical success by endoscopy avoiding surgery was achieved in $90.6 \%$ of cases.

\section{Introduction}

Endoscopic submucosal dissection (ESD) is widely used in many specialized centers to treat superficial neoplasia in the colon because this is associated with less morbidity than conventional surgery [1]. Lesions in close proximity to the appendiceal orifice
(L-PAO) were initially considered unresectable by endoscopic means due to high risk of incomplete resection, perforation, and acute appendicitis [2, 3]. Nevertheless, it has been demonstrated by Jacob et al. to be feasible by ESD with the exception of deep invasion of the appendiceal orifice (Toyonaga's type 3) [4]. Recently, we reported a new strategy of "Double-Clips and 
569 lesions in the colon and rectum resected by ESD

between January 2018 and April 2019

(Lyon: 266, Limoges: 202, Bordeaux: 101)

Excluded:

494 lesions not located in the cecum.

75 cecal lesions resected by DCT-ESD

(Lyon: 48, Limoges: 23, Bordeaux: 4)

Excluded: 43 lesions not in contact with

appendiceal orifice

32 lesions in contact with appendiceal orifice resected by DCT-ESD, included in the study

(Lyon: 23, Limoges: 5, Bordeaux: 4)

- Fig. 1 Flowchart of the study.

rubber band Traction" (DCT-ESD) allowing resection of lesions deeply invading the appendix [5]. In the current study, we focused on the consecutive DCT-ESD performed for L-PAO and aimed to evaluate the feasibility and the outcomes according to Toyonaga's classification of appendiceal invasion [4].

\section{Patients and methods}

\section{Patients}

We conducted a retrospective analysis of a prospective collected database of consecutive 569 colorectal ESD performed in three French centers from January 2018 to April 2019. Seventy-five cecal lesions treated by DCT-ESD were identified and among them, we excluded 43 lesions that were not in contact with appendiceal orifice. A total 32 lesions in contact with appendiceal orifice resected by DCT-ESD were eligible and included for the study ( $\mathbf{F i g . 1}$ ). Chromoendoscopy was used to assess the appropriate indication of endoscopic resection, according to the European Society of Gastrointestinal Endoscopy guidelines [6]. All patients received a pre-procedure consultation with a clear explanation of ESD and its possible adverse events including the potential requirement of salvage surgery.

\section{Inclusion}

Colorectal lesions touched the appendiceal orifice featured noninvasive characteristics (Sano II or IIla, Kudo IV or Vi) and whose size required ESD to be removed en bloc were included. All lesions resected were described according to Toyonaga's classification, which included type 1: touched the appendiceal orifice; type 2: invaded the orifice partially; type 3 : invaded the orifice deeply (no normal mucosal is seen inside the orifice) and we also included lesions deeply invading the area of previous appendectomy and called this type 3a ( $\mathbf{F i g}$. 2). Type 0 lesions were excluded as these are not in closed contact with appendiceal orifice. All included patient was contacted by email

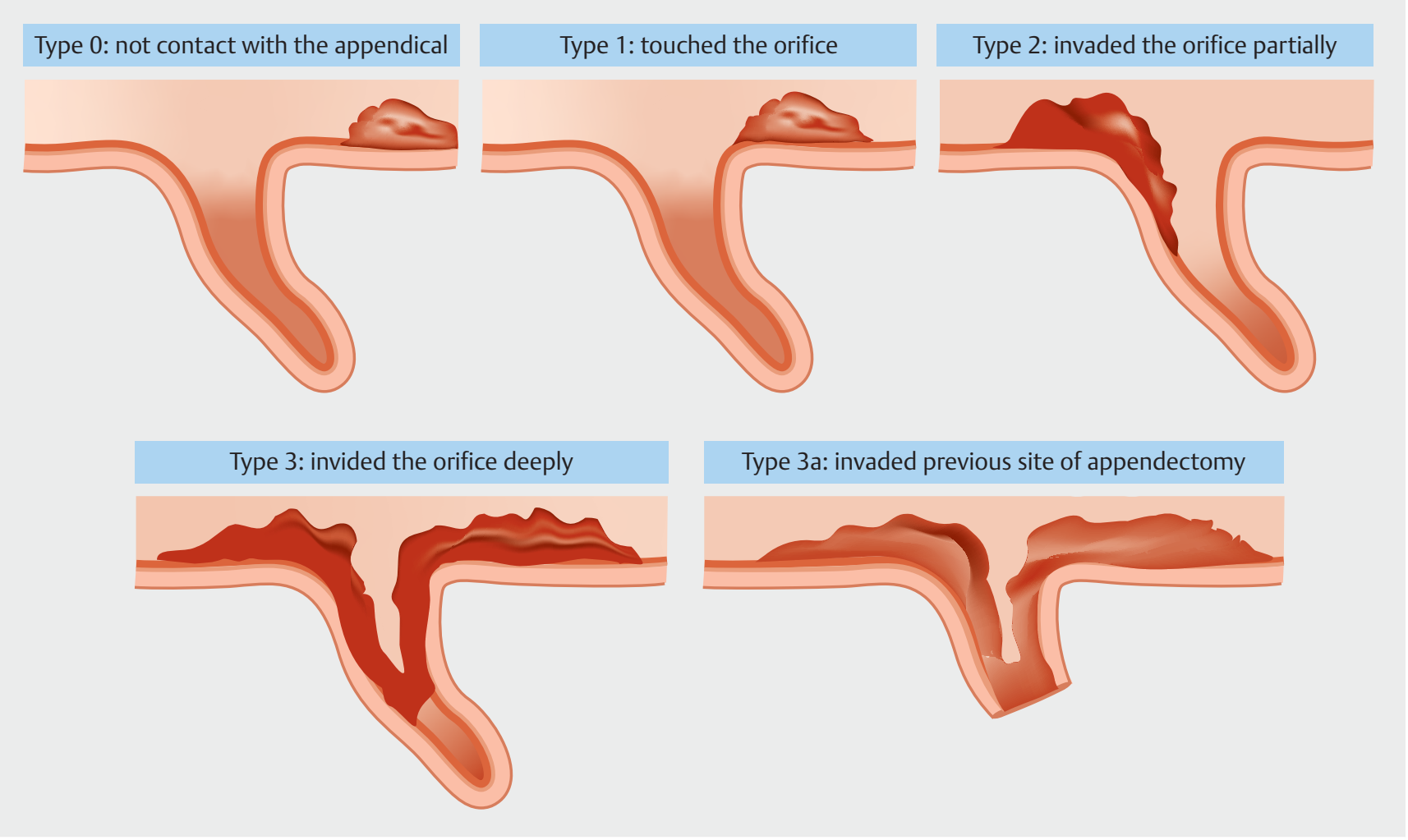

Fig. 2 Lesion classification according to Toyonaga's classification with additional type 3a in case of previous appendectomy. 
or came for a consultation for clinical follow-up at 3 months post-procedure. In case of incomplete resection, a 3-month follow-up colonoscopy was scheduled. For others, follow-up colonoscopy was indicated depending on their histological result, according to French recommendation.

\section{Endoscopic procedure}

The ESD procedure was performed under general anesthesia with intubation, and colon inflation was done using $\mathrm{CO}_{2}$. We used therapeutic colonoscopes (PCF 190L, PCF 190T, or PCF190TL; Olympus, Tokyo, Japan) with a $4 \mathrm{~mm}$ distal cap attached (D-201-11304, Olympus). For all procedures, we used Dualknife Jet knife (KD-655U, Olympus), Resolution 360 clip (Boston scientific, Boston,USA) and ERBE VIO 200D or 300 D electrosurgical system (Erbe, Tübingen, Germany). Hydroxyethylamidon (HEA) mixed with indigo carmine was used for initial submucosal injection.

\section{Strategic approach}

All lesions were resected by DCT-ESD according to the following strategy which was described by Jacques et al ( $\mathbf{F i g . 3 , ~}$ > Fig. 4) [7] First, after submucosal injection, a complete circumferential incision and deep trimming were performed. Second, we placed the traction system (composed of two clips and a rubber band). Depending on the level of insufflation, it was possible to adjust manually the degree of countertraction to facilitate the submucosal dissection and to pull the lesion out from the orifice gradually. Then, the submucosa was dissected as much as possible. For lesions deeply invading the appendiceal orifice without previous appendectomy (type 3), dissection was performed as deeply as possible in the orifice to achieve a complete submucosal dissection of the whole appendix, with two different strategies. While arriving at the base of the orifice, if the submucosal space could not be visualized despite sufficient submucosal injection and the counteraction force, the operator decided to cut the appendiceal mucosa circumferentially as close as possible to base of the appendix, to finish the procedure ( $\triangleright$ Fig. $\mathbf{3 c}$ "). In this situation, the margin of the specimen was not certain. Nevertheless, a complete resection passing through the submucosal fibrosis at the base of the orifice was

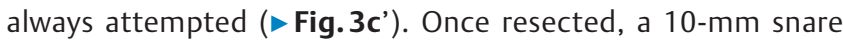
was used to remove the clip from the opposite colonic wall to retrieve the specimen ( $\nabla \mathbf{F i g . 3 d}$ ). The specimen was then stretched on a cork board and sent to the pathologist ( $\triangleright$ Fig.5).

\section{Primary outcome}

The primary outcome was the proportion of technical success defined as En Bloc resection exclusively with ESD (no snaring) and $R 0$ resection after histological examination (free margins).

\section{Secondary outcome}

Secondary outcomes were proportion of curative resections, defined by histologically En Bloc resection with free margins according to the European recommendations [6]; the frequency of adverse events related to the ESD procedure as well as additional surgery for complications or incomplete resection; the duration of procedure, defined as the time from injection to specimen fall; and the frequency of systematic follow-up. The degree of submucosal fibrosis according to the appearance of submucosal space during submucosal injection with Indigo carmine [8].

\section{Statistical analysis}

Fischer's exact test was used to compare outcomes according to Toyonaga's type of L-PAO. Independent sample $t$-test was used to compare mean number of hospitals stay. $P<0.05$ was considered to be statistically significant. All analyses were conducted using SPSS software (Version 23, IBM Corp., Armonk, New York, United States).

\section{Ethical concern}

All patients gave their operating consent before receiving DCTESD for L-PAO, and this study was approved by the ethics committee of the Hospices Civils de Lyon (Lyon, France).

\section{Results}

A total 32 patients (32 lesions) underwent DCT-ESD for L-PAO, $56.3 \%$ were male and the mean age was 67 years.

\section{Lesion characteristics}

According to Toyonaga's classification, 11 lesions (34.4\%) were type 3, 11 were type 3a (34.4\%), seven were type 2 (25.0\%), and three were type 1 (10.7\%). More than two-thirds of lesions $(68.8 \%, n=22)$ of lesions had F2 submucosal fibrosis. Macroscopic and histological characteristic of lesions are described in $>$ Table 1.

\section{Technical results}

DCT-ESD was possible in all cases with success of traction positioning in all cases $(n=32,100 \%)$. En bloc resection was achieved in all cases $(n=32,100 \%$; Table 2$)$. The median duration of procedure was 47 minutes (range: 10-230), and the median lesion size was $35 \mathrm{~mm}$ (range: 10-70). Submucosal dissection reached the base of the orifice with complete dissection of the whole appendix was achieved in all cases of type $3 a$ $(n=11,100 \%)$ and 1 case of type $3(n=1,9.1 \%)$.

\section{Primary endpoint}

Histologically confirmed complete resection with free margins (R0) was obtained for $90.6 \%$ lesions $(n=29)$. In total, 3 resections were R1 with a lateral margin in contact with only lowgrade dysplasia. One of the patients was diagnosed with a synchronous invasive adenocarcinoma in the descending colon and surgeons decided to perform an additional appendectomy during the colectomy for the second invasive lesion; no residual adenoma was found on the appendectomy specimen. Other two patients underwent a control colonoscopy at 3 months without local recurrence. The overall curative resection rate was $90.6 \%$ ( $\triangleright$ Table 2 ). 


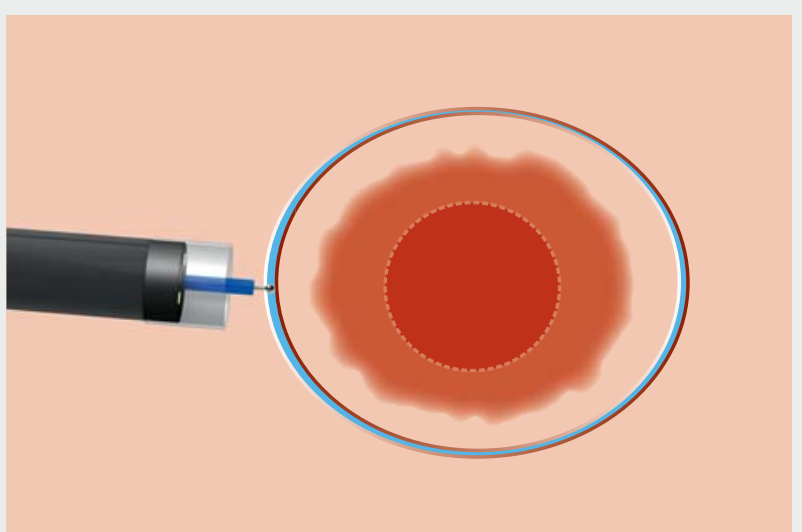

a

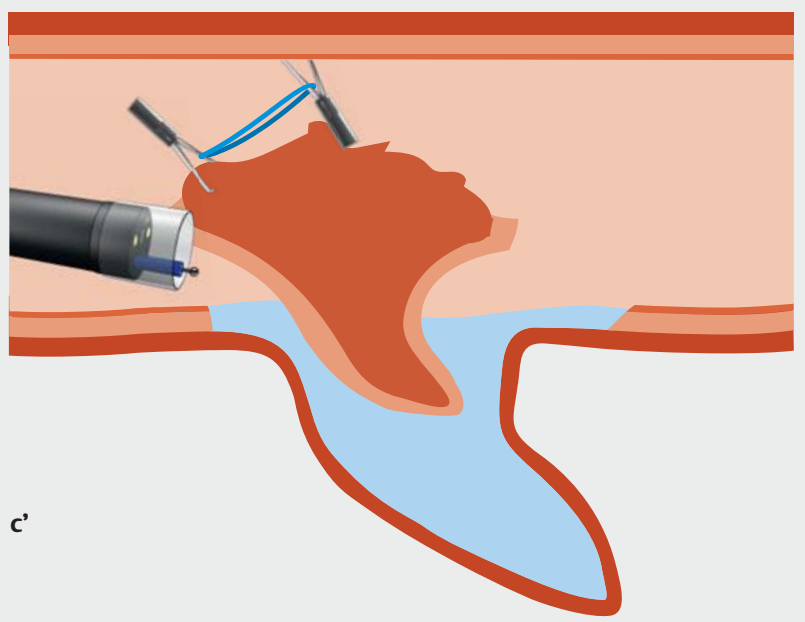

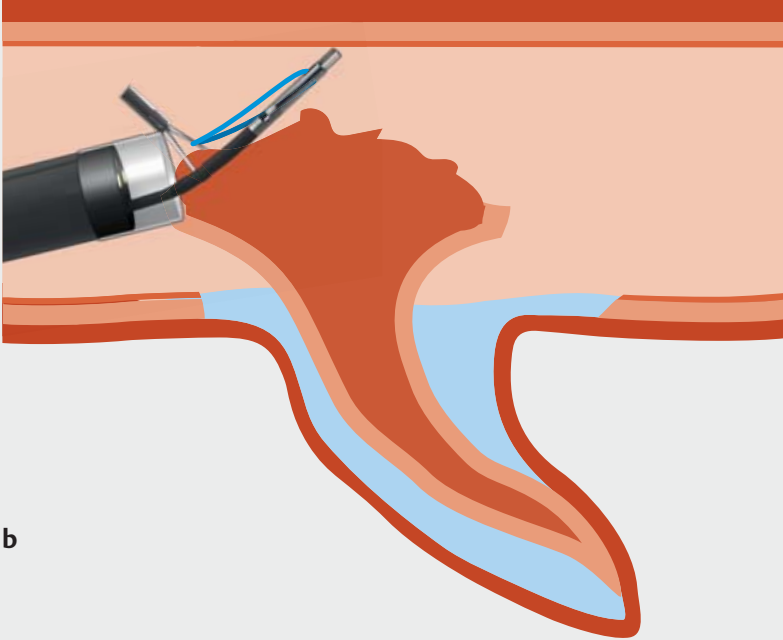

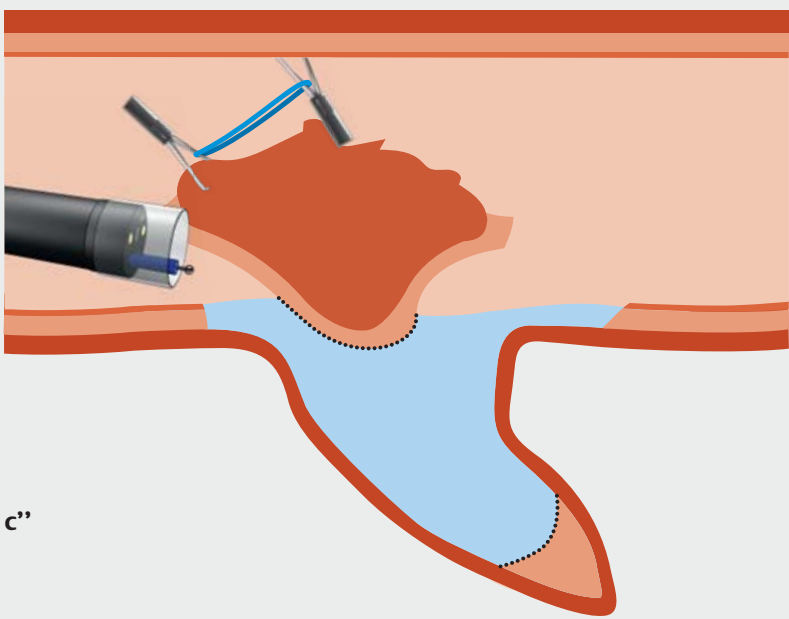

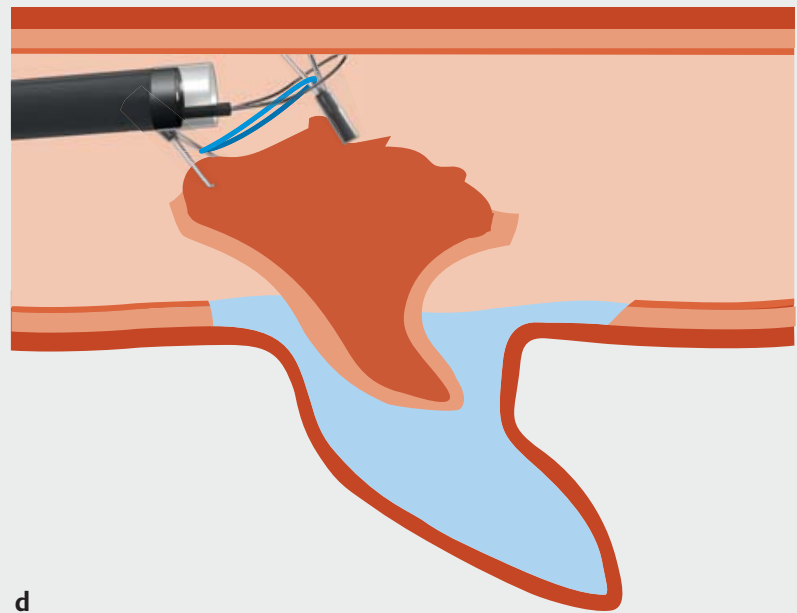

- Fig. 3 DCT-ESD strategy. a Complete circumferential incision and deep trimming were performed around the appendiceal area to make a mucosal flap with large free margins. b The first clip (Resolution 360, Boston Scientific, Boston, Massachusetts, United States) grasping a rubber band was inserted through the working channel and was fixed on the edge of the mucosal flap. Immediately after, a second clip was used to grasp the rubber band stretching and fixing it to a fold of the opposite colonic wall. The elasticity of rubber band created more or less traction according to the degree of inflation to adjust the traction force. As a result, the tumor was pulled out gradually from the orifice, which allowed stretching the submucosal layer facilitating deep dissection. c' If possible, we cut all submucosal space through the base of the orifice. c" If submucosal space could not be seen despite enough injection, and deep progression became impossible, we cut the appendiceal mucosa circumferentially to achieve resection. $\mathbf{d}$ Finally, we used $10-\mathrm{mm}$ snare to remove the clip attached to the opposite colonic wall to withdraw the resected lesion. 

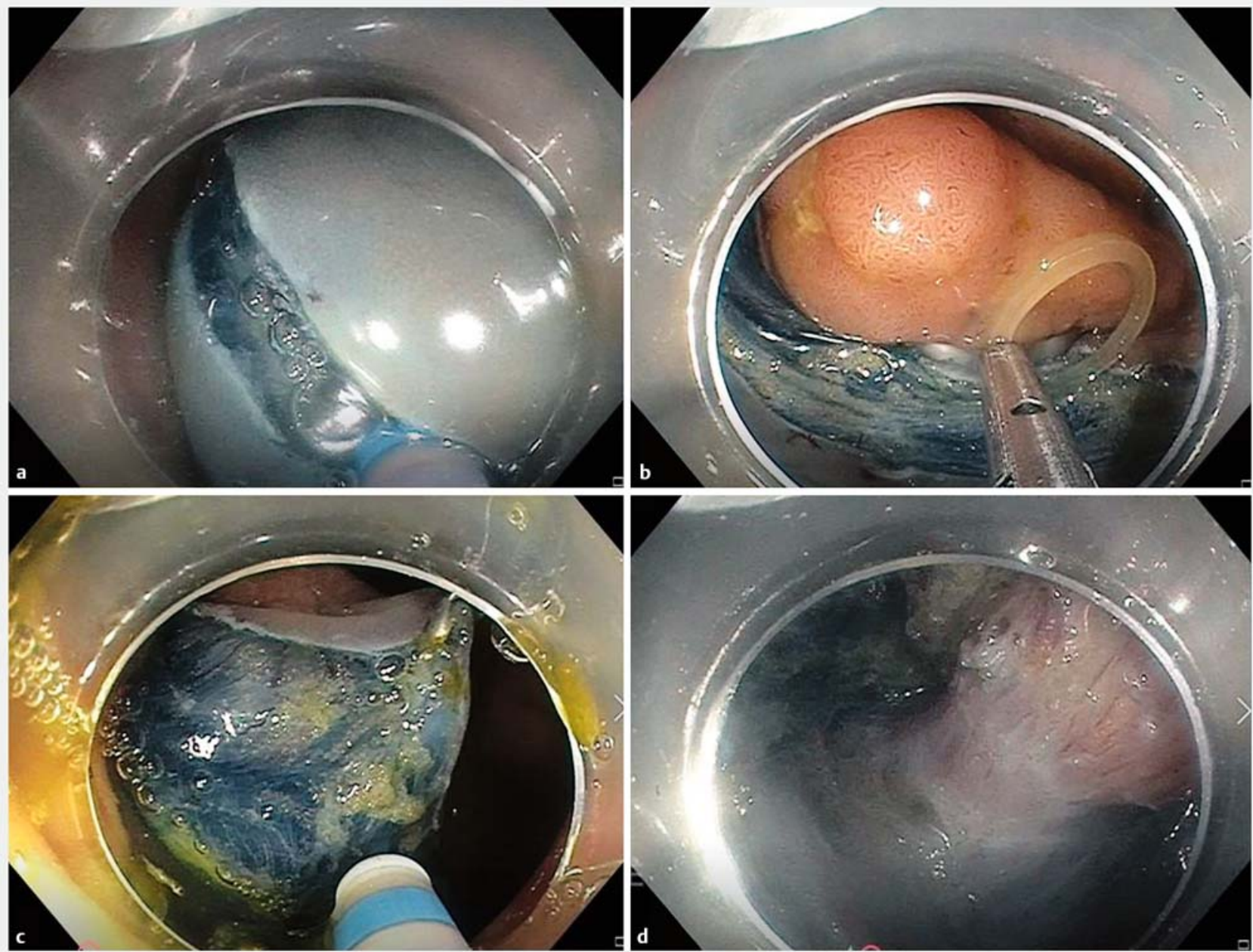

- Fig. 4 Strategy of DCT-ESD for L-PAO. a Circumferential incision and deep trimming. b First clip with rubber band attached on the edge. c Dissection under traction after fixation of the second clip grasping rubber band on the opposite wall. $\mathbf{d}$ Deep dissection following appendix submucosa.
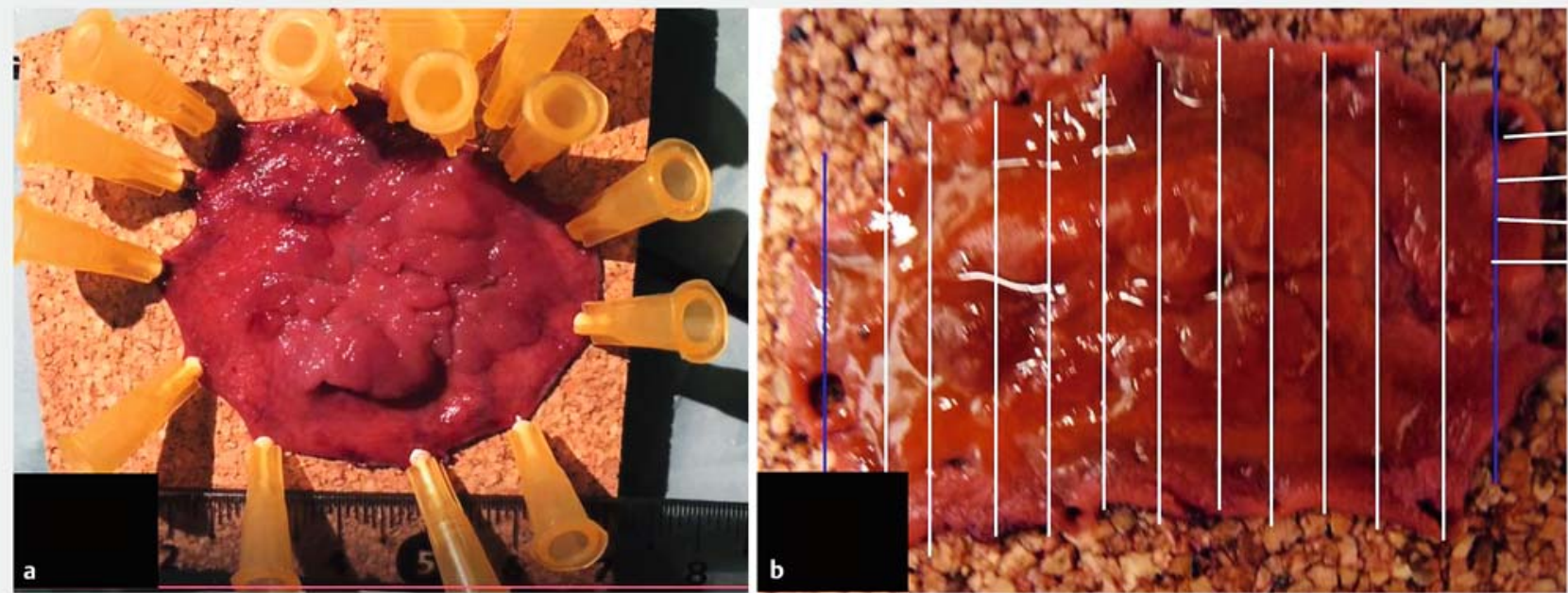

Fig. 5 Aspect of the stretched specimen and usual slicing by pathologists. a The specimen was then stretched on cork board, and we measured its large diameter size. $\mathbf{b}$ The specimen was sliced in $2-\mathrm{mm}$ pieces and analyzed by expert pathologists. 
- Table 1 Characteristics of the 32 patients and lesions.

\begin{tabular}{|c|c|}
\hline Characteristics & $\begin{array}{l}\text { Total population, } \\
\mathrm{n}=32\end{array}$ \\
\hline Mean age, year \pm SD (range) & $67 \pm 11(42-88)$ \\
\hline Male sex, n (\%) & $18(56.3 \%)$ \\
\hline Previous appendectomy & $11(34.4 \%)$ \\
\hline \multicolumn{2}{|l|}{ Lesion classification, n (\%) } \\
\hline - Type 1 & $3(9.4 \%)$ \\
\hline - Type 2 & $7(21.9 \%)$ \\
\hline - Type 3 & $11(34.4 \%)$ \\
\hline - Type 3a & $11(34.4 \%)$ \\
\hline \multicolumn{2}{|l|}{ Macroscopic Morphology, n (\%) } \\
\hline - LST-G homogenous & $13(40.6 \%)$ \\
\hline - LST-G with macronodule $>1 \mathrm{~cm}$ & $1(3.6 \%)$ \\
\hline - LST-G with central depression & $1(3.1 \%)$ \\
\hline - LST-NG homogenous & $1(3.1 \%)$ \\
\hline - Serrated lesion & $11(34.4 \%)$ \\
\hline - Polyp & $5(15.6 \%)$ \\
\hline \multicolumn{2}{|l|}{ Submucosal fibrosis, n (\%) } \\
\hline - F0 & $2(6.3 \%)$ \\
\hline - $F 1$ & $8(25.0 \%)$ \\
\hline - $F 2$ & $22(68.8 \%)$ \\
\hline - Lesion size, median (range), mm & $35(10-110)$ \\
\hline - Procedure time, median (range), minutes & $47(10-230)$ \\
\hline - Number of clips, mean \pm SD (range) & $2 \pm 2(1-7)$ \\
\hline - Hospital stay, mean \pm SD (range), nights & $2.6 \pm 2(2-12)$ \\
\hline \multicolumn{2}{|l|}{ Histology, n (\%) } \\
\hline - High-grade dysplasia & $10(31.3 \%)$ \\
\hline - Low-grade dysplasia & $8(25.0 \%)$ \\
\hline - Intramucosal adenocarcinoma & $1(3.1 \%)$ \\
\hline - Submucosal cancer $<1000 \mu \mathrm{m}$ & $1(3.1 \%)$ \\
\hline - Serrated lesion without dysplasia & $11(34.4 \%)$ \\
\hline - Serrated lesion with low-grade dysplasia & $1(3.1 \%)$ \\
\hline
\end{tabular}

LST-G, laterally spreading tumor-granular; SD, standard deviation.

\section{Adverse events}

Per-procedure, 11 perforations occurred (34.4\%) and were immediately and successfully closed with clips (mean number of clips 2; range: 1-7). Among 10 diminutive perforations $(<5 \mathrm{~mm})$, one patient developed postoperative mild peritonitis resolved with antibiotics without further surgery. One larger perforation complicated by synchronous post-operative peritonitis and acute appendicitis despite the endoscopic clo- sure underwent salvage surgery without stoma. Delayed perforation with peritonitis occurred in one patient; this also required surgery. Despite the surgery for adverse events, endoscopic curative resection was achieved in all three patients. Neither delayed bleeding nor death occurred. According to Toyonaga's classification, all perforations leading to surgery occurred for type 3 lesions without previous appendectomy ( Table 2).

Patients were discharged after a mean 2.6 nights in hospital (range: 2-12 nights); the mean length of hospital stay was longer for those with complication-related additional surgery ( $n=2,9.5$ nights) than those who did not $(n=30,2.1$ nights, $P=0.0001)$ In total, three patients underwent additional surgery $(9.4 \%)$ including two cases related to a complication and one case related to $\mathrm{R} 1$ resection; overall post-ESD complication-related surgery was $6.3 \%$. All patients were clinically followed-up 3 months later by consultation or by email. No delayed adverse events such as acute appendicitis was reported.

\section{Discussion}

The current study found that DCT-ESD allowed Enbloc resection for all appendiceal lesion although more than $90 \%$ of cases were resected in a curative manner without additional surgeries.

Other teams had previously focused on these appendix lesions, with good results in terms of curative resections $[3,9$, 10]. However, only Jacob et al. accurately described the degree of invasion of the appendicular orifice; the authors included lesions up to type 3 a and report that a caecum lesion not touching the appendicular opening can be resected like any other coIonic lesion, while the risk of incomplete resection and complication increases considerably as the invasion into the appendix increases [4]. Therefore, studies that do not describe invasion in the appendix should be interpreted with caution as they mix procedures associated with very different risks. It is of note that in the study reported by Jacob et al. deep invasions of the appendicular opening (type 3 ) were even considered contraindications to endoscopic resection and excluded from the study. Nonetheless, they have shown that for type 1 and 2 lesion, endoscopic resection by conventional ESD with the help of gravity traction was achievable in expert hands. Based on our experiences, patient rotation during procedure was often required to create gravity traction and sometime more than one rotation was needed, making the task difficult for the team as the patient was always intubated, which could prolong procedure time. Herein, all type lesions (1, 2, 3 and 3a) were successfully resected by DCT-ESD with much shorter procedure time, compared to those of conventional ESD reported by Jacob et al. [4]. Furthermore, despite the technical difficulty induced by the underlying scarring in type 3 a lesions, the procedure was more frequently successful and less frequently associated with complications (in particular perforation) than on the native appendix (type 3). It is therefore probably necessary to distinguish these from type 3 lesions and therefore add a subtype to Toyonaga's classification. In these subtypes with prior appendectomy, Full-thickness resection device (FTRD) is an alterna- 
- Table 2 Outcomes in different type of L-PAO according to Toyonaga's classification.

\begin{tabular}{|c|c|c|c|c|c|c|}
\hline & Type 1 & Type 2 & Type 3 & Type 3a & Overall & $P$ value $^{1}$ \\
\hline \multicolumn{7}{|l|}{ Effectiveness outcomes n/N (\%) } \\
\hline En Bloc resection rate & $3 / 3(100 \%)$ & $7 / 7(100 \%)$ & $11 / 11100 \%)$ & $11 / 11(100 \%)$ & $32 / 32(100 \%)$ & NA \\
\hline R0 resection rate & $3 / 3(100 \%)$ & $6 / 7(85.7 \%)$ & $9 / 11(81.8 \%)$ & $11 / 11(100 \%)$ & $29 / 32(90.6 \%)$ & 0.266 \\
\hline Curative resection & $3 / 3(100 \%)$ & $6 / 7(85.7 \%)$ & $9 / 11(81.8 \%)$ & $11 / 11(100 \%)$ & $29 / 32(90.6 \%)$ & 0.266 \\
\hline \multicolumn{7}{|l|}{ Adverse events $\mathrm{n} / \mathrm{N}(\%)$} \\
\hline Per-operative perforation & $1 / 3(33.3 \%)$ & $3 / 7(42.9 \%)$ & $6 / 11(54.5 \%)^{2,3}$ & $1 / 11(9.1 \%)$ & $11 / 32(34.4 \%)$ & 0.123 \\
\hline Delayed perforation with peritonitis & 0 & 0 & $1 / 11(9.1 \%)$ & 0 & $1 / 32(3.6 \%)$ & 0.344 \\
\hline Acute appendicitis & 0 & 0 & $1 / 11(9.1 \%)^{4}$ & 0 & $1 / 28(3.6 \%)$ & 0.344 \\
\hline Additional surgery & 0 & 0 & $3 / 11(27.3 \%)^{5,6}$ & 0 & $3 / 32(9.4 \%)^{5,6}$ & 0.033 \\
\hline $\begin{array}{l}{ }^{1} \text { Comparison between Type } 3 \text { without pr } \\
{ }^{2} \text { One patient developed delayed local pe } \\
{ }^{3} \text { One patient developed postoperative p } \\
{ }^{4} \text { One patient with per-operative perfora } \\
{ }^{5} \text { One patient had R1 resection with a syn } \\
\text { related surgery rate is } 6.3 \% \text { ( } 2 \text { cases). } \\
{ }^{6} \text { Postoperative complication related surs }\end{array}$ & $\begin{array}{l}\text { Dendectomy an } \\
\text { is, resolved with } \\
\text { tis requiring ad } \\
\text { eveloped peritor } \\
\text { ous invasive ade } \\
\text { te is } 6.3 \% \text { ( } 2 \text { cas }\end{array}$ & $\begin{array}{l}\text { hers, using Fish } \\
\text { tt antibiotics } \\
\text { nnal surgery } \\
\text { and postopera } \\
\text { arcinoma in the }\end{array}$ & $\begin{array}{l}\text { exact test } \\
\text { appendicitis } \\
\text { cending colon and ur }\end{array}$ & rwent additionals & gery, Postoperative & nplication- \\
\hline
\end{tabular}

tive solution [11] without the risk of appendicular mucocele and acute appendicitis that exist for non-operated appendix FTRDs.

On the other hand, for lesions that deeply invade the appendicular orifice on an unoperated appendix (type 3), there was a trend towards more frequent perforations, closed endoscopically in most cases, and leading to surgery in less than a quarter of cases. The therapeutic strategy is therefore open for discussion for type 3 lesions, although $75 \%$ of patients eventually undergo complete endoscopic resection without further surgery, the remaining quarter will undergo surgical management as a matter of urgency for a complication or at a distance for incomplete resection. The other option would be to propose the patient directly for surgery, but in cases where the lesion extends away from the appendix on the cecum, a simple enlarged appendectomy is not sufficient, as it may not be complete by cutting into the caecal lesion. Ileocecal resection or a right colectomy for a benign lesion due to appendicular invasion should not, however, be proposed as segment colectomy is associated with $20 \%$ morbidity and $0.5 \%$ mortality [12]. FTRD resection could theoretically be a good idea, but by closing the appendix, the risk of acute appendicitis is $25 \%$ [13-15] and it has recently been reported that this may also cause translocation of adenoma tissue into peritoneum in case of incomplete resection [16]. In this context, submucosal dissection with traction can be proposed after informing the patient of the risks of secondary surgery.

The study does have limitations, such as its retrospective nature, although all consecutive cases are presented in an exhaustive manner, taking into account invasion and including lesions with deep invasion of the appendiceal orifice (type 3 ). The second limitation is that no systematic early control colonoscopy was performed when the resection was complete with lateral and deep safety margins according to histological analysis; however, these patients will be followed-up at 1 or 3 years according to French recommendations depending on histology.

\section{Conclusion}

In conclusion, colorectal neoplastic lesions invading the appendicular opening should no longer be considered as contraindications to endoscopic resection. Describing the degree of invasion into the orifice with lesion classification such as those reported by the Japanese team of Toyonaga should be helpful for endoscopist to select resection strategies and stratify the risk of incomplete resection as well as adverse events. DCT-ESD allowed us to treat all type of L-PAO effectively even when the invasion occurs deeply in the appendix, with a less invasive manner compared to open surgery and less financially burdensome, compared to FTRD. However, morbidity increases with invasion into the appendix, so a shared discussion with the patient should be done to opt a suitable treatment.

\section{Competing interests}

The authors declare that they have no conflict of interest.

\section{References}

[1] Saito $Y$, Uraoka T, Yamaguchi Y et al. A prospective, multicenter study of 1111 colorectal endoscopic submucosal dissections (with video). Gastrointest Endosc 2010; 72: 1217-1225

[2] Fujishiro M, Yahagi N, Kakushima $\mathrm{N}$ et al. Outcomes of endoscopic submucosal dissection for colorectal epithelial neoplasms in 200 consecutive cases. Clin Gastroenterol Hepatol 2007; 5: 678-683 
[3] Horimatsu T, Fu K-I, Sano Y et al. Acute appendicitis as a rare complication after endoscopic mucosal resection. Dig Dis Sci 2007; 52: 1741-1744

[4] Jacob H, Toyonaga T, Ohara Y et al. Endoscopic submucosal dissection of cecal lesions in proximity to the appendiceal orifice. Endoscopy 2016; 48: 829-836

[5] Utzeri E, Jacques ], Charissoux A et al. Traction strategy with clips and rubber band allows complete en bloc endoscopic submucosal dissection of laterally spreading tumors invading the appendix. Endoscopy 2017; 49: 820-822

[6] Pimentel-Nunes P, Dinis-Ribeiro M, Ponchon T et al. Endoscopic submucosal dissection: European Society of Gastrointestinal Endoscopy (ESGE) guideline. Endoscopy 2015; 47: 829-854

[7] Jacques J, Charissoux A, Bordillon P et al. High proficiency of colonic endoscopic submucosal dissection in Europe thanks to countertraction strategy using a double clip and rubber band. Endosc Int Open 2019; 7: E1166-E1174

[8] Matsumoto A, Tanaka S, Oba S et al. Outcome of endoscopic submucosal dissection for colorectal tumors accompanied by fibrosis. Scand J Gastroenterol 2010; 45: 1329-1337

[9] Binmoeller KF, Hamerski CM, Shah JN et al. Underwater EMR of adenomas of the appendiceal orifice (with video). Gastrointest Endosc 2016; 83: 638-642
[10] Song EM, Yang H-J, Lee HJ et al. Endoscopic resection of cecal polyps involving the appendiceal orifice: a KASID multicenter study. Dig Dis Sci 2017; 62: 3138-3148

[11] Lupu A, Jacques J, Rivory J et al. Hybrid endoscopic submucosal dissection using a full-thickness resection device allows en bloc resection of a large adenoma deeply invading the appendix. Endoscopy 2018; 50: E296-E298

[12] Le Roy F, Manfredi S, Hamonic S et al. Frequency of and risk factors for the surgical resection of nonmalignant colorectal polyps: a population-based study. Endoscopy 2016; 48: 263-270

[13] Bronzwaer ME, Bastiaansen BA, Koens L et al. Endoscopic full-thickness resection of polyps involving the appendiceal orifice: a prospective observational case study. Endosc Int Open 2018; 6: E1112-E1119

[14] Schmidt A, Bauerfeind P, Gubler C et al. Endoscopic full-thickness resection in the colorectum with a novel over-the-scope device: first experience. Endoscopy 2015; 47: 719-725

[15] Wedi E, Orlandini B, Gromski M et al. Full-thickness resection device for complex colorectal lesions in high-risk patients as a last-resort endoscopic treatment: initial clinical experience and review of the current literature. Clin Endosc 2018; 51: 103

[16] Dumoulin FL, Gorris DG, Berger $S$ et al. Full-thickness resection with an over-the-scope device: possible translocation of adenoma tissue in a case of an incomplete resection at the appendix. Endosc Int Open 2018; 6: E622-E624 